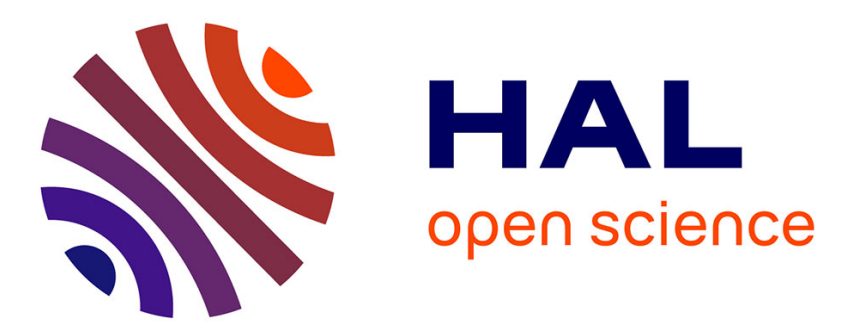

\title{
RESONANT DYNAMICAL REGIMES IN A LONG JOSEPHSON JUNCTION MODULATED BY A LARGE-SCALE SPATIAL INHOMOGENEITY
}

J. Fernandez, G. Reinisch

\section{- To cite this version:}

J. Fernandez, G. Reinisch. RESONANT DYNAMICAL REGIMES IN A LONG JOSEPHSON JUNCTION MODULATED BY A LARGE-SCALE SPATIAL INHOMOGENEITY. Journal de Physique Colloques, 1989, 50 (C3), pp.C3-163-C3-168. 10.1051/jphyscol:1989325 . jpa-00229466

\section{HAL Id: jpa-00229466 https://hal.science/jpa-00229466}

Submitted on 1 Jan 1989

HAL is a multi-disciplinary open access archive for the deposit and dissemination of scientific research documents, whether they are published or not. The documents may come from teaching and research institutions in France or abroad, or from public or private research centers.
L'archive ouverte pluridisciplinaire $\mathbf{H A L}$, est destinée au dépôt et à la diffusion de documents scientifiques de niveau recherche, publiés ou non, émanant des établissements d'enseignement et de recherche français ou étrangers, des laboratoires publics ou privés. 
RESONANT DYNAMICAL REGIMES IN A LONG JOSEPHSON JUNCTION MODULATED BY A LARGE-SCALE SPATIAL INHOMOGENEITY

\author{
J.C. FERNANDEZ and G. REINISCH \\ Observatoire de Nice, BP. 139, F-06003 Nice Cedex, France
}

Résumé - Dans une jonction Josephson longue, spatialement inhomogène, on peut obtenir des régines dynamiques périodiques et stables pour un fluxon, sous l'action d'un champ externe oscillant et d'un faible courant. Ces régimes montrent une marche de voltage particulière sur la caractéristique courant-tension. Une telle signature suggère de faire une vérification expérimentale de ces accrochages de phase.

Abstract - A fluxon, in a long and spatially inhomogeneous Josephson junction, undergoes stable periodic dynamical regimes, under the action of an external field and a weak bias current. These regimes show a special step in the I-V characteristics. Such an effect suggests the experimental verification of these phaselock regimes.

\title{
1 - PRELIMINARY ON JOSEPHSON EFFECT
}

Physically, a Josephson junction is a couple of superconductor metal layers built as a "sandwich" about thin layer of insulator (see figure 1). With respect to the observed phenomena, a Josephson junction is a system in which a quantum-mechanical quantity like the phase difference $\phi$ of the wave functions describing the electrons, has a macroscopic meaning; this phase $\phi$ is related to the current $I$ and to the voltage $V$.

The two basic Josephson equations are:

$$
\begin{aligned}
& I=I_{0} \sin \phi, \\
& \frac{d \phi}{d t}=\frac{2 \pi}{\phi_{0}} V,
\end{aligned}
$$

where $I$ is the tunnel suppercurrent, $I_{0}$ is the maximum Josephson current depending on the junction geometry, the material and the temperature, $t$ is the time, $\phi_{0}$ is a flux quantum equal to $h / 2 e\left(\sim 2.10^{-15} \mathrm{~V}\right.$.s. $)$, and $V$ is the voltage (i.e. the potential difference across the junction). Equations (1) and (2) describe the dymamics of the so-called short junctions (this will be precised below).

Even with $V=0$ which is equivalent to $d \phi / d t=0$, we may have $\phi=C t e \neq 0$, which corresponds to a non zero current. This presence of such a current at zero voltage is called the direct Josephson effect.

Moreover, if $V=C t e \neq 0$, we obtain an alternative supercurrent whose frequency is close to $484 \mathrm{MHz} / \mu \mathrm{V}$. This corresponds to the ac Josephson effect.

Introducing the Josephson penetration length

$$
\lambda_{j}=\sqrt{\frac{\hbar}{2 e \mu_{0} I_{0} d}},
$$

with $d=\lambda_{L 1}+\lambda_{L 2}+t^{*}$ in which $\lambda_{L i}$ represent the London depth $[1]$ of the $i^{\text {th }}$ superconductor and $t^{*}$ the barrier height, and coupling the Maxwell equations with the Josephson equations give

$$
\frac{\partial^{2} \phi}{\partial x^{2}}-\frac{1}{\bar{c}^{2}} \frac{\partial^{2} \phi}{\partial t^{2}}-\frac{1}{\lambda_{j}^{2}} \sin \phi=\frac{\mu_{0} d}{R_{0}} \frac{\partial \phi}{\partial t},
$$

where $R_{0}$ is the quasi particle resistance.

In the static case, $\phi$ obeys

$$
\begin{aligned}
& \partial^{2} \phi \\
& \partial x^{2}
\end{aligned}=\frac{1}{\lambda_{j}^{2}} \sin \phi \quad,
$$

and if $\phi \ll 1$, then $\phi(x)=A e^{x / \lambda_{j}}+B e^{-x / \lambda_{j}}$ 


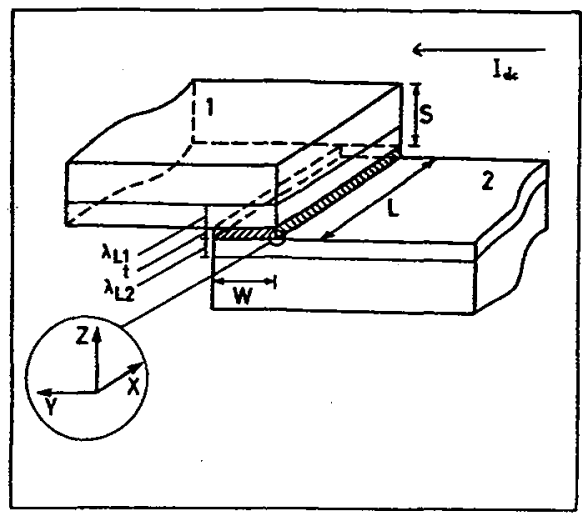

Fig. 1 - Typical geometry of a Josephson junction (here an overlap junction). Typical material is $S n-S n_{x} O_{y}-$ $S n$. Typical dimensions are $S \sim 4000 A ; \lambda_{L i} \sim 500 A ; t^{*} \sim 10-40 A ; L \sim 1 \mathrm{~mm} ; L / \lambda_{j} \sim 5-40 ; W / \lambda_{j} \sim 20 \mu m$. The temperature is around $4^{\circ} K, \bar{c} \sim$ few $\%$ of $c$ and $\omega_{j} \sim 10^{9}-10^{11} \mathrm{~Hz}$. After S. Pagano.
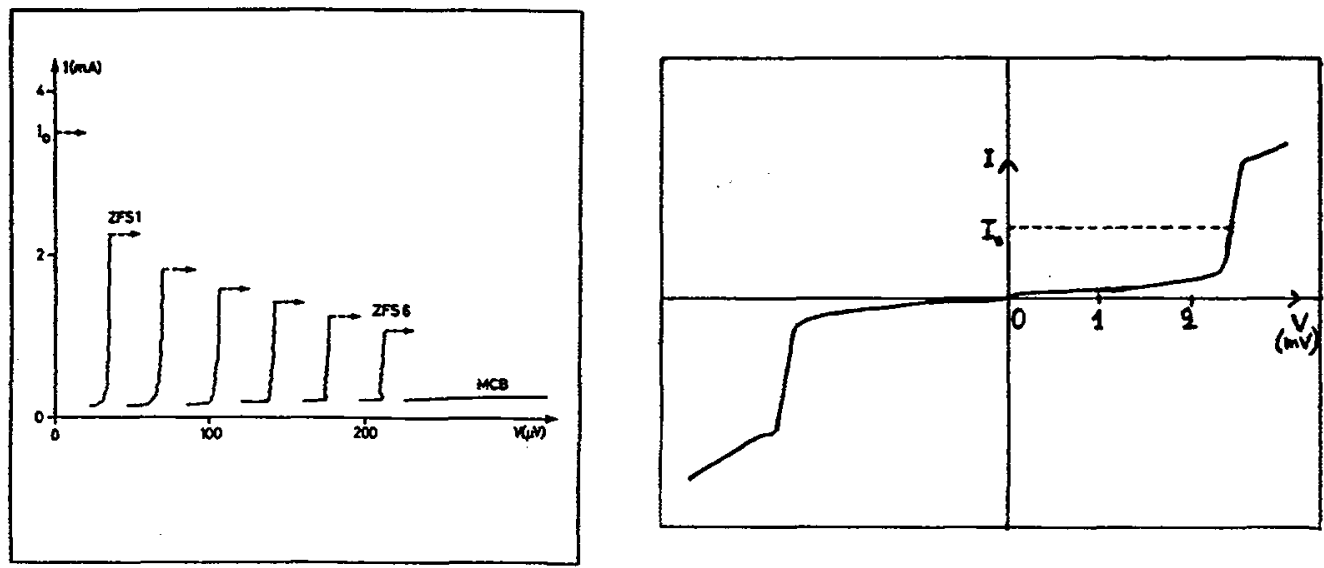

Fig. 2a - Typical experimental I-V characteristic of a Josephson junction. $I_{0}$ is the Josephson critical current. The sample is $\mathrm{Nb}-\mathrm{N} b_{x} \mathrm{O}_{y}-\mathrm{Pb}$ junction of overlap geometry. After $\mathrm{S}$. Pagano.

Fig. $2 b$ - Typical low voltage region of the I-V characteristic of a Josephson junction. The sample is the same as for Figure 2. Six ZFSs are visible. After S. Pagano.

This shows that $\lambda_{j}$ is the typical distance over which the phase changes.

Taking into account this typical length, we get a more precise definition of a "short" and a "long" junction, corresponding respectively to a junction whose length is respectively smaller or bigger than $\lambda_{j}$.

If we also introduce the Swihart velocity $\bar{c}=\sqrt{\frac{i^{*}}{\varepsilon \varepsilon_{0} \mu_{n} d}}$ and the plasma frequency $\omega_{j}=\frac{\bar{c}}{\lambda_{j}}$, and if we normalise the lengthes to $\lambda_{j}$ and the times to $\omega_{j}^{-1}$, equation (4) becomes

$$
\phi_{t t}-\phi_{x x}+\sin \phi=-\alpha \phi_{t}
$$

which is a damped normalized sine-Gordon equation.

\section{2 - PRELIMINARY ABOUT SINE GORDON PHYSICS}

The most interesting solution of the sine-Gordon equation

$$
\phi_{t t}-\phi_{x x}+\sin \phi=0,
$$


with respect to Josephson physics is the kink:

$$
\phi_{S}(x)=4 \tan ^{-1} \exp (-\sigma x)
$$

where $\sigma$ defines the polarity. Depending on the sign of $\sigma$ we have a kink $(\sigma=-1)$ or an antikink $(\sigma=1)$. The Lorentz invariance of the equation (6) allows a family of one parameter solutions deduced from (7) whose velocity $v$ is limited to 1

$$
\phi_{S}(x, t)=4 \tan ^{-1} \exp (-\sigma \zeta)
$$

with

$$
\zeta=\gamma(x-v t) ; \gamma=\frac{1}{\sqrt{1-v^{2}}}
$$

We emphasize that the direct proportionality between the voltage $V$ and $\phi_{\ell}$ implies a proportionality of the voltage on the product $\sigma v$. Linking the Josephson physics to the sine Gordon one, we point out that, in term of soliton $\lambda_{j}$ corresponds to the soliton width. The equation (6) can be derived from an hamiltonian density

$$
h=\frac{1}{2} \phi_{t}^{2}+\frac{1}{2} \phi_{x}^{2}+1-\cos \phi
$$

and we can calculate the energy corresponding to a soliton

$$
H=\int h\left(\phi_{S}\right) d x=8 \gamma
$$

\section{3 - ADDING AN INHOMOGENEITY TO THE JUNCTION}

In the case of a large scale inhomogeneous long Josephson junction, the partial differential equation describing the phase can be written like

$$
\phi_{t \ell}-\phi_{x x}+\left[1+\frac{1}{4} V(x)\right] \sin \phi=0,
$$

where $\mathrm{V}(\mathrm{x})$ represent the spatial inhomogeneity or what is often called here below the potential well.

We have shown $[2]$ that, if the potential well is smooth enough, i.e. if its typical scale is large compared to the soliton width, the energy of the soliton in the low energy limit $V \ll 1$ can be written as

$$
H=8 \gamma+\sqrt{\frac{1+\frac{1}{4} V(Y)}{1-\dot{Y}^{2}}} V(Y) \quad,
$$

where $Y$ represent the soliton position. If, in addition we suppose that the soliton behaviour is restricted to non relativistic dynamic we get

$$
H \sim \frac{1}{2} 8 \dot{Y}^{2}+V(Y)=C t e=E
$$

which correspond to a Newton law.

If we take a potential well like $\chi x^{2}$ we recover for the non-relativistic soliton the dynamic of an harmonic oscillator.

Assuming now that we allow relativistic dynamics we have shown $[3]$ that the modulated kink like solution of equation (11) can be written

$$
\phi(x, t)=4 \tan ^{-1} \exp \{-\sigma k(t)(x-Y(t))\} \quad,
$$

with

$$
k(t)=\sqrt{\frac{1+\frac{1}{4} V(x)}{1-Y^{2}}}
$$




\section{4 - PhenOMENOLOGICAL Deschiption}

Assume first an homogeneous finite long Josephson junction with free ends, i.e. with boundary conditions

$$
\phi_{x= \pm L / 2}=0
$$

If we consider a kink with an initial velocity $v_{0}$ in such a junction without any damping (which is an unrealistic case) the dynamics correspond to a shuttle regime at velocity $v_{0}$. Each time the kink reaches an end it is changed into a reflected antikink (i.e. its polarity $\sigma$ changes its sign) which moves at velocity $-v_{0}$. In this periodic regime we get a non-zero voltage (averaged over the time) since the product $\sigma v_{0}$ remains constant. We must mention also that each time the kink or antikink reaches an end of the junction, it emits an electromagnetic signal. The frequency $\omega_{e}$ of this emission basically depends on the velocity $v_{0}$ of the kink and the length $L$ of the junction: $\omega_{e}=v_{0} / 2 L$.

Considering now the same initial condition in the same junction but taking into account the resistive effect (i.e. the damping) of the junction, the kink will begin to experience a shut tling regime with a decreasing velocity, until it is stopped. So, in a finite time we will recover a static kink and then the average voltage will be zero. We must point out another possible behaviour for the kink; the reflection at one end is equivalent to a collision between the real kink and a virtual antikink, and we know $[4]$ that in presence of damping it exists a threshold value of the velocity of the colliding kink, under which the kink and the (virtual) antikink will be bunched together, giving birth to a breather which will also be damped, giving itself birth to phonons which finally damp out. The value of this threshold depends only on the damping coefficient $\alpha$ (see equation 5 ).

Considering now a kink at rest as the initial condition, in a junction without damping, but biased by a direct current (dc), the soliton will be accelerated and will reach its limit velocity which is 1 in the normalized units. We then recover a shuttle regime giving voltage and emission.

If we now consider both damping and bias, the soliton will reach a limit velocity $v_{l}$ which is the signature of the energy balance between the input energy trough the dc term $I_{d c}$ and the output energy through the damping term. This limit velocity reads:

$$
v_{l}=\left[1+\left(\frac{4 \alpha}{\pi I_{d c}}\right)^{2}\right]^{-1 / 2} .
$$

We get also in this case a shuttling regime giving voltage and emission at frequency $\omega_{l}=v_{l} / 2 L$.

Consider now an inhomogeneous junction, and more precisely a potential well like $V(x)=a(1-\operatorname{sechbx})$ with $b \ll 1$ and $L \gg 1$. This system has an eigen frequency $\Omega_{b}$ due to the presence of the potential well. Indeed, linearizing the solution around a kink, we can extract an eigenvalue problem [5]. Suppose we start with a soliton at rest at the bottom of the well and we add an external if field, the equation is then

$$
\phi_{t t}-\phi_{x x}+\left(1+\frac{1}{4} V(x)\right) \sin \phi=\varepsilon \cos (\Omega t)-\alpha \phi_{t}
$$

We have then the possibility to excite the soliton with the external field. If $\Omega \neq \Omega_{b}$ we recover small "internal" oscillation of the kink. The slope of the kink oscillates but the kink remains located about the bottom of the well.

If we choose $\Omega$ closer to the value $\Omega_{b}$ we recover, in addition to this "internal" oscillations, a small amplitude oscillation of the position of the kink in the potential well. We have transformed a part of the external energy into kinetic energy for the soliton.

If now $\Omega=\Omega_{b}$ we are in a resonnance situation and most of the external input energy is transformed into kinetic energy for the kink. The kink experiences large amplitude oscillations. It may then happen (depending on the depth of the potential well) that the kink goes out of the well and begin to shuttle in the junction, being reflected in an antikink at the end. There is a competition between this shuttle driven-damped regime and the resonnant-trapped regime. In order to be sure that the kink movement is not stopped in the homogeneous part of the junction by the damping we also add a direct current to the equation (18).

The question arising now: does a periodic regime exist, when taking into account this competition ?

We have numerically shown that for given values of the direct current (which is taken as the control parameter) we obtain such regimes as limit cycles for the corresponding dynamical system (see figure 3) [6]. 


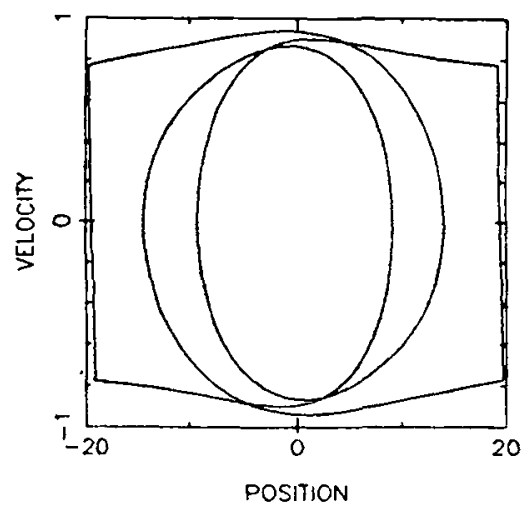

Fig. 3 - The asymptotic phase-locked cycle obtained either by a direct numerical simulation of the PDE (20) or by the numerical solution of the ODE system (21-25) where: $L=40 ; a=20 ; b=0.095 ; \varepsilon=0.1 ; \Omega=0.049 ; \alpha=$ $0.01 ; I_{d c}=0.02$. The cycle was obtained at time $t$ up to 20000 .

\section{5 - MODELISATION OF THE PARTIAL DIFFERENTIAL EQUATION}

Inserting the ansatz (15) into the following PDE

$$
\phi_{t \ell}-\phi_{x x}+\left(1+\frac{1}{4} V\right) \sin \phi=-\alpha \phi_{t}+\varepsilon \cos (\Omega t)+I_{d c} \quad,
$$

and projecting the resulting equation on the soliton Goldstone mode, then noting that the $k$ oscillations are at high frequency while the $Y$ ones are at low frequency, which allows to arverage the motion of $k$ over a period of $Y$, we get the following ordinary differential equation (ODE) for the dynamics of the kink-like solution [3]:

$$
\ddot{Y}=\frac{1}{8 k}\left\{\left(1-\dot{Y}^{2}\right)\left[2 \pi \sigma\left(I_{d c}+\varepsilon \cos (\Omega t)-8 \alpha k \dot{Y}\right]-\frac{a b}{k} \frac{\sinh (b Y)}{\cosh ^{2}(b Y)}\right\},\right.
$$

with

$$
k=\left[\frac{1+\frac{1}{4} V(Y)}{1-Y^{2}}\right]^{1 / 2} .
$$

One can recognize in equation (20) both ( $\mathrm{dc}$ and ac) driving terms whose effect depends on the sign of $\sigma$, the damping term slowing down the kink and the last term which describes the newtonian action of the potential well on the particle (see equation 13).

Actually this'ODE describes the soliton dynamics governed by equation (19) except for the boundary conditions which we still have to modelise. As said before, free ends boundary conditions for the PDE (19) described by equation (16) can be viewed like collision between a kink and a virtual soliton of opposite polarity. We know that there exists a phase shift, an additionnal loss of energy, a change of polarity and a possible death of the soliton due to a trapping between the particle and its virtual anti-particle during the reflexion [4]. The phase shift is given by

$$
\delta=-2 \sqrt{1-v^{2}} \ln |v|
$$

The lost energy during the reflexion is

$$
\delta H=-2 \pi^{2} \alpha
$$

The change of polarity consists only on replacing $\sigma$ by $-\sigma$.

Finally the soliton can be killed when its velocity is less than $v_{t h}$ defined by

$$
\frac{1}{2} 8 v_{t h}^{2}<2 \pi^{2} \alpha
$$


Numerical simulations have been done with this ODE (20-21) and the boundary conditions modelized by (22-24). They show extremely good agreement with what is numerically obtained by the PDE (19) [6]. It is very surprising to see that choosing only one degree of freedom, namely $Y$, we recover the dynamic described by the PDE which depends on an infinite degrees of freedom. This is probably related to the very high stability of the kink solution of sine Gordon, which admits an adiabatic, WKB-like modulation of its parameters when the properties of the propagating medium slowly change.

\section{6 - SOME INTERESTING PROBLEMS}

If we vary the control parameter $I_{d c}$, keeping all other parameters constant, it seems that we obtain a route to chaos through period doubling. It is interesting to see that the "soliton" then behaves chaotically in time, still preserving its spatial coherence $[7]$

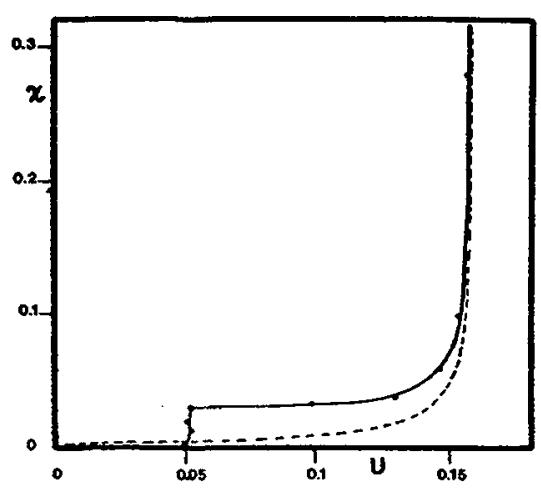

Fig. 4 - Typical I-V characteristic obtained for phase-locked regimes. The dashed curve corresponds to the unperturbed ZFS.

Another interesting problem concerns the possible phase-lock of an homogeneous junction driven with an rf external field without any bias $[8]$. This shows cyles of half the external period giving a possibility of a frequency doubler. We can for example imagine an array $[\mathbf{9}]$ of such coupled junctions which could both increase the emitted power and the output frequency by successive doubling.

The phase lock in an inhomogeneous junction and its signature on the I-V characteristic (see figure 4) suggest to build up the experiment in order to check this effect. Building up the potential well which is a controlled spatial modulation of the critical current does not seem an easy task, neither to check a posteriori the shape of this modulation.

This work was financially supported by the EEC (Economic European Community) contract ST2-0267-J-C. We thank our colleagues N. Flytzanis, M.J. Goupil, R. Grauer, S. Pagano, N.F. Pedersen, S. Pnevmatikos and M. Taki for stimulating and interesting discussions.

\section{REFERENCES}

1/ Barone A., Paterno G. "Physics and applications of the Josephson effect", John Wiley, NY, (1982).

/2/ Fernandez J.C., Leon J.P., Reinisch G., Phys. Rev. B 27, 5817; (1983).

/3/ Fernandez J.C., Goupil M.J., Legrand O., Reinisch G., Phys. Rev. B 34, 6207, (1986).

/4/ Pedersen N.F., Samuelsen M.R., Welner D, Phys. Rev. B 30, 4057, (1984).

/5/ Leon J.P., G. Reinisch, J.C. Fernandez, Phys. Rev. B 27, 5817, 1983 and Fernandez J.C., Passot T., Politano

H., Reinisch G., Taki M., Phys. Rev. B 37, 7342, 1988.

/6/ Reinisch G., Fernandez J.C., Flytzanis N., Taki M., Pnevmatikos S. Submitted to Phys. Rev. B.

/7/ Fernandez J.C., Goupil M.J. Reinisch G. In preparation

/8/ Fernandez J.C., Grauer R., Reinisch G., submitted to Phys. Rev. Lett.

19/ Cirillo M., J. Appl. Phys. 58, 3217, (1985) and 60, 338, (1986).

/10/ Pagano S., Phd Thesis, Lynby, Danemark, 1987. 